$$
\text { DOE/EW/12823-T6 }
$$

\title{
Three Dimensional Characterization and Archiving System
}

\author{
Richard L. Sebastian (richard_sebastiant(G)ail.cre.corn); 703-719-9200) \\ Robert Clark (bob_clark isail_crc.com); 703-719-9200) \\ Phitip Gallman (phil_gallman(- mail.crc.com); 703-719-9200) \\ James Gauditeault (jm_gaudreaslt (4 mail.crc.com); 703-719-9200) \\ Richard Mosehauer (fichard_uxosehauer@mailcre.com); 703-719-9200) \\ Dana Simonson (dana_simonson_omail.crc.com); 709-719-9200) \\ Anthony Slotwinski (tony_slotwitski(G mait.cre.com); 703-719-9200) \\ Colensan Research Corporation \\ 6551 Loisdale Coutrt, Saite 800 \\ Springfield, VA 22150
}

\author{
Eugene Achter(617-938-065i) \\ George Janvis(617-938-0651) \\ Tlyemedics Detection, Inc. \\ 220 Mill Road \\ Chelnisford, MA 01824
}

\author{
Peter Grifiths (208-885-6552) \\ Nathar Chaffin(208-885-6552) \\ Ian Lewis(208-885-6552) \\ Department of Chemistry \\ University of Idaho \\ Moscow, ID 83844
}



\subsection{Introduction}

The Three Dimensional Characterization and Archiving System (3D-ICAS)' is being developed as a remote system to perform rapid in situ analysis of hazardous organics and radionuclide contamination on structural materials. Coleman Research and its subcontractors, Thermedics Detection, Inc. (TD) and the University of Idaho (UI) are in the second phase of a tirree phase program to develop 3D-ICAS to support Decontamination and Decommissioning (D\&D) operations. Accurate physical characterization of surfaces and the radioactive and organic is a critical D\&D task. Surface characterization includes

${ }^{1 R}$ esearch sponsored by the U.S. Department of Energy's Morgantow Technology Center, tonder contract DEAC31-93MC30176 with Coleman Research Corporaiton, 6551 Loisdale Court, Springfield, VA 22150; fax 703-719-9229. identification of potentially dangerous inorganic materials, such as asbestos and transite.

Real-time remotely operable characterization instrumentation will significantly advance the analysis capabilities beyond those currently employed. Chemsical analysis is a primary area where the characterization process will be improved. Chemical atalysis plays a vital role throughout the process of decontarnination. Before clean-up operations can begin the site must be characterized with respect to the type and concentration of contaminaxts, and detailed site mapping must clarify areas of both high and low risk. During remediation activities chemical analysis provides a means to measure progress and to adjust clean-up strategy. Once the clean-up process has been completed the results of chemical 


\section{DISCLAIMER}

This report was prepared as an account of work sponsored by an agency of the United States Government. Neither the United States Government nor any agency thereof, nor any of their employees, make any warranty, express or implied, or assumes any legal liability or responsibility for the accuracy, completeness, or usefuiness of any information, apparatus, product, or process disclosed, or represents that its use would not infringe privately owned rights. Feterence herein to any specific commercial product, process, or service by trade name, trademark, manufacturer, or otherwise does not necessarily constitute or imply its endorsement, recommendation, or favoring by the United States Government or any agency thereof. The views and opinions of authors expressed herein do not necessarily state of reflect those of the United States Government or any agency thereof. 


\section{DISCLAIMER}

Portions of this document may be illegible in electronic image products. Images are produced from the best available original document. 
analysis will verify that the site is in compliance with federal and local regulations.

The development of the field operable in situ analysis capability of the 3D-ICAS witl restilt in a significantly improved capability to perform analyses for trace organic compounds and radionuclides by providing real-time quantitative results on-site. This will greatly improve the effectiveness of Department of Energy (DOE) response to identified sites by streamlining the entire beginning-to-end process from the initial contamination surveys, through monitoring the progress of site restoration efforts, and determining when regulatory standards have been met. The field operable remotely directed analysis instrumentation of 3D-ICAS will have great impact in terms of:

- Improving the quality and efficiency of site clean-np activities

- Reducing health and safety risks to the survey workers

- Reducing the associated cost and time required for remediation

- Reducing waste generation

\subsection{The Approach}

The 3D-ICAS system robotically conveys a multisensor probe near the surfaces to be inspected. The seasor position and orientation are monitored and controlled using coherent laser radar (CLR) tracking. The CLR also provides 3D facility maps which establish a 3D "world view" within which the robotic sensor system can operate.

The 3D-ICAS fills the need for high speed automated organic analysis by means of a gas chromatographs-mass spectrometer sensor which can process a sample, without direct contact, accomplișing detection and fine grain analysis of regulatory concentrations [EPA 1987 spill cleanup policy: 1 itg/
$10 \mathrm{~cm}^{2}$ for high use interior building surfaces) in approximately one minute. This compares with traditional GC-MS laboratory analysis methods which involves sample preparation and waste generation, and take hours per sarnple for analysis. The 3D-ICAS GC-MS sensor extracts volatile organics directly from contaminated surfaces without sample removal, then uses multiple stage focusing to accomplish bigh time resolution insertion into a high speed gas chromatograph. Detection and additional discrimination are provided by a final stage time-of-flight mass spectrometer. This high speed process replaces sample collection and transport and hours of solution preparation before injection into an ordinary GC-MS which typically bas a 45 minute nun time.

The radionuclide sensors of the 3D-ICAS multisensor probe combines $a, B$, and $g$ counting with energy discrimination on the a channel. This sensor combination identifies and quantifies isotopes of specific DOE interest of uranium, plutonium, thorium, technetium, nepumitum, and americium to regulatory levels in approximately one minute.

The Molecular vibrational spectrometry (MVS) sensor of the multisensor probe is used to characterjze substrate material such as concrete, wood or asbestos. The surface composition information provided by the MVS can be used to provide estimates of the depth of contamination and to optimize the analysis performance of the other contamination detection sensors. In addition, the materials composition information, combined with the surface geometry maps provided by the coherent laser radar, will provide a more complete three dimensional world view to be usec to plan and execte robotic D\&D operations.

The 3D-ICAS will scan operator designated areas with a full sensor set or a selected subset at a designated sample density. The 3D-ICAS will plan and Jaser track the sensor trajectory to assure efficient, close sensing without surface contact. 
The 3D-ICAS sensor output and contamination analysis along with CLR position information will be available for real-time monitoring inmediately after each one minute sample period. After a high bandwidth functions such as servo loops, gas chromography, and laser radar transmission and teception.



Figure 3-1. 3D-ICAS Phase II Conffguration

surface mapping operation is completed, 3D-ICAS will provide three dimensional displays showing contours of detected contaminant concentrations. The 3D-ICAS will further provide pentanent measurement data and contaminant level archiving, asstring data integrity and allowing straightforward regulatory review of the characterization process before and after D\&D operations.

The 3D-ICAS Phase II demonstration configuration is shown in Figure 3-1.

Figure 3-2 shows the interconnection of the major subsystems. The links are a combination of serial for slow speed command and control, TCP/P network for file based data file storage and comorntnications, and dedicated antog and digital links for
The Integrated Workstation communications with the TCP/IP network based link to the CLR control computer. This link provides all bartware control functions and database access for the Integrated Workstation.

The CLR computer serves as a focal point for hardware control. Serial links are used between the CLR computer and the Multisensor Probe electronics control computer to provide control and status information. Data from the Muitisensor Probe is stored directly on a TCP/AP network drive. Serial links are afso used to control the robot arm position. The 3D-ICAS Phase $I$ demonstration multisensor probe is shown in Figure 3-3. 




Figure 3-2. 3D-ICAS Phase II Demonstration Interfaces


HUF SIRE



Figure 3-3. Block Diagram of the Phase I Demonstration Multisensor Probe

\subsection{The Development Program}

The 3D-ICAS is being developed in a three phase 32 month program. The current Phase II in- cletes integration of the GC and MS subsysterns, integration of the multisensor probe with the robot arm and the coherent laser radar tracker. The Phase I3D-ICAS demonstration, involving contamination 
surface mapping with a CLR guided, roboticalyy maneuvered multisensor probe, will take place in November, 1995.

The Phase III development program will integrate and test the total system in a mobile field prototype and demonstrate the systems at one or more DOE sites.

A stustantial part of the Phase II effort is the integration of the 3D CLR mapping and robotic sensor positioning and tracking capability with the sensor data output in a common coordinate frame so that $3 \mathrm{D}$ archiving of contamination surveys can take pface. This paper will not discuss detailed system integration issues, but will discuss areas of developing technical performance of the GC-MS sensor, the radionuclide sensor, the MVS sensor, and the CLR 3D mapper and 6DOF end effector tracker which are of interest to the 3D-ICAS user.

\subsection{The GC-MS Sensor Subsystem}

The 3D-ICAS GC subsystem consists of an automated surface sampling, preconcentration and transport subsytem, high speed GC separation capability, (greater than 100 peaks per minute and fast detector electronics. These aspects of the high speed GC technology were successfully demonstrated in Phase I.

The high speed GC system consists of a focusing module and a chromatography module. The focusing module consists of a short length of GC column inside its metallic sheath, coupled to a cooling source. The focusing module is maintained at low temperature while the sample is introduced in a stream of air or carrier gas. At low temperature, the analytes are retained on the stationary phase of the GC column in the focusing module.

After sample focusing, the sample is injected by rapid healing of the focusing module. Sampie injection is achieved with tight spatial conerence in less than 50 milliseconds, which helps to produce sharp chromatographic peaks. The GC system uses two sequential focusing stages, to provide additional selectivity and tighter injection onto the chromatograph. After the sampie is injected from the second focusing stage, the GC column is then heated through a rapid, tightly-controlled temperature program. Controlled heating rates in excess of $1000 \mathrm{C}$ per second can be achieved. With this technology, the accessible heating rate is mo longer a limitation to chromatographic performance. The speed and resolution of the chromatography is determined by processes such as carrier gas flow rate, and molecular diffusion over very short distances, which are inherently fast.

A GC breadboard test system for the current effort was developed to contain all the required elements. The breadboard system provided the cryofocusing elements ("cold spots"), sample switch" ing valves, temperature controller, and analog and digital electronics for high speed temperature programming. Externally-mounted experimental GC columns were interfaced to the breadboard chassis. The high speed temperature programming electronics were modified extensively to accommodate columin lengths up to 7 meters, and chromatographic elution times up to 40 seconds.

In Pbase I, the high speed GC was integrated with a time-of-flight mass spectrometer and analysis software development to achieve rapid, definitive quantitative measurement of trace level orgaric contamination in the presence of a complex background, as can be expected in DOE facilities.

A block diagtam of the High Speed Gas Chromatograph/Mass Spectrometer Sensor is shown in Figure 3-4.

A time-of-flight mass spectrometer (TOFMS) system was chosen based on its ability to main- 




Figure 3-4. Block Diagram of the High Speed Gas Chromatograph/Mass Spectrometer

tain a high data throughput. Early on it was determined that effective High Speed GC-MS would require a detector system capable of 10 millisecond or less response time. The time-of-flight system is the only mass analyzer capable of such a data rate.

The TOF-MS is operationally simple and reliable. All mass spectrometers have some form of interface to the outside world, all require bigh vacuum chambers, all require ionization source and all require defectors. In general what differentiates mass spectrometers is the technology for mass analysis. For 3D-ICAS, a very fast detector is require to fully utilize the capabilities of the high speed GC technology and hence be capable with the surface contamisation mapping function. A time-of-flight mass analyzer was chosen because it can provide full mass spectral scan (to $600 \mathrm{amu}$ ) for every ionization event. Other types of mass spectrometers canno generate full mass spectra ar these high rates. The full mass spectra is necessary for identification of the unknown components of a sample through library searching. Addjtionally, existing TOF technology will allow high repetition scan rates for inplementing high duty cycle, high speed operation of the TOF MS detector when coupled to the High Speed GC.

Ionization events were generated by the MS at a $10 \mathrm{KHz}$ repetition rate, 128 events were summed into one data point along the GC axis. This GC sampling period is sufficient to clearly distinguish the $G C$ peak and deconvolute peaks, if necessary. For a GC peak that is 100 milliseconds foll wide at half height (FWHM) approximately 1000 full scan mass spectra are recorded across the full width at half maximum. A signal to noise enhancement advantage can be achieved by summing the mass spectra into discrete sampling windows. For the current system's GC peaks on the order of 100 milliseconds wide a data sample period of approximately 10 milliseconds will clearly define the GC peaks. Other mass analysis technologies cannot provide mass spectra at these high repetition rates.

One of the most critical parts of a GC-MS is the interface between the systems. The interface 
plays the important role of accommodating the pressure drop from the $G C$ column exit to the MS ionization source and eariching the concentration of the analyte in the carrier gas after passing through the interface into the mass spectrometer. The requirements for a GC-MS interface are high transfer efficiency, no ingact on the GC separation, no degradation of compounds, no preferential removal of compounds or chemical functional groups. Of all the techniques available the molecular beam interface was chosen as optimal for this fast analysis system.

The directed beam of molecules has an advantage over conventional therral, diffusive methods for introduction of the molecules into the ionization source. The concentration of molecules within the volume of space that the electron gun molecular beam crosses is close to a maximum. Thus, probability of ionization per unit time is increased, thereby ircreasing sensitivity.

\subsubsection{GC/MS Processing}

A block diagram of the GC/MS and radionuclide sensor processor is shown in Figure 3-5. The overall function of these components are to acquire the time-of-flight MS data as a function of GC elution time, control the GC operation, control the radionuclide detectors for data collection and processing. The acquired data from the GCMS sensor is processed into mass spectra and total ion chromatogram. The GCMS data are processed through a National Institutes of Standardis (NIST) Mass Spectral Database, response factor calibration and sample classification algorithms for further data reduction.

The electronics technology for the HSGCMS tata acquisition and processing were the high speed transient digitizer, high speed data transfer bus and high speed digital signal processors (DSP). The



Figure 3-5. Block Diagram of the GC/MS and Radionuclide Sensor 
MS events occur on a nanosecond time scale. A mass spectral scale from 35 to 600 anmu requires approximately 40 microseconds of data To achieve a mass spectral resolution of one at 600 annu requires 5 to 10 nanosecond time discrimination.

\subsubsection{GCMS Test Results}

The tests were carried out using the breadboard GC-MS to optinize the performance charac- temperature to $280 \mathrm{C}$ in 17 seconds then hold at 280 C tor 5 seconds. The overall cycle time was 30 seconds. About 35 peaks are resolved, the detected conpounds list indicates that chlorinated biphenyls compounds have been identified. Shown in Figure 3-7 is a portion of the list of compounds identified through the NIST library search during the analytical rum. The list contains the retention time the search confidence level and the name of the compound identified. The list will be expanded to include the chemi-



Figure 3-6. Total Lon Chromatogram for a Mixture of PCBs Analyzed using the High Speed Gas Chrortatograph/Mass Spectrometer

teristics and demonstrate overall function. The function included sampling from surface, GC separation and MS detection, identification and quantification.

The results of a typical GCMS man are shown in Figure 3-6. A $1 \mu \mathrm{L}$ injection of a mixture of PCBs (arochiors 1221,1248 , and 1254) was used in this test. The chronatographic conditions were as follows: 6 meter DB-5 and helium carrier gas at $100 \mathrm{~cm} /$ sec. The temperature program was as follows: room cal classification and the amount of compound detected as the software is completed in the project. It is expected that the detection sensitivity of this instrumentation will meet or exceed the regulatory levels of $1 \mu g / 10 \mathrm{~cm} 2$ for high use interior building surfaces (EPA Spill Cleanup Policy, 1987). Figuse 3-8 shows the mass spectrum used in the library search for the compound detected at 24.8 seconds. A comparison of Figure 3-7 and 3-8 indicates that the detected compound was identified as trichlorobiphenyl 
c.



Figure 3-7. Mass Spectrum Measured at 24.800 Seconds

\begin{tabular}{|c|c|c|}
\hline 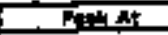 &  &  \\
\hline 24:00 and & 79,574 & 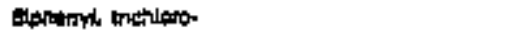 \\
\hline $25 a 12$ mas & $40.67 \%$ &  \\
\hline $25312 \mathrm{mu}$ & $\begin{array}{l}21.77 \% \\
17.04 \% \\
17.75 \% \\
11.1 \% \\
6.55 \%\end{array}$ &  \\
\hline $75000 \mathrm{mo}$ &  &  \\
\hline 25180 mat & 7.324 &  \\
\hline $26152 \mathrm{mt}$ &  &  \\
\hline $2048 \mathrm{gm}$ & $04.27 \%$ & Stoheny, Bentestere. \\
\hline $2 \cos 2$ mis & $\operatorname{ag} 05$ &  \\
\hline 29980 ats & $\begin{array}{l}19.45 \% \\
15.80 \% \\
17.64 \% \\
10.14 \% \\
0.19 \%\end{array}$ &  \\
\hline 27352 the & $52.25 \%$ & 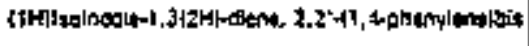 \\
\hline $27602 \mathrm{mu}$ & $6.96 \%{ }^{\prime}$ &  \\
\hline
\end{tabular}

Figure 3-8. A Portion of the HSGC-MS Processed Data 
Tonal Ion Chromatogran

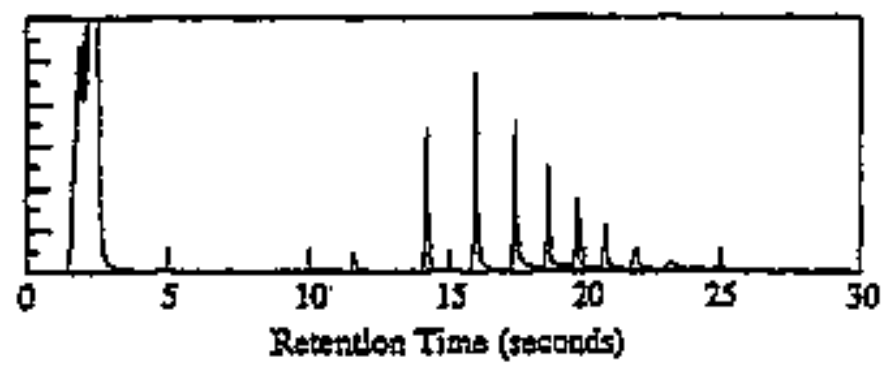

Measured Mass Spectrum at 15.9580 seconds

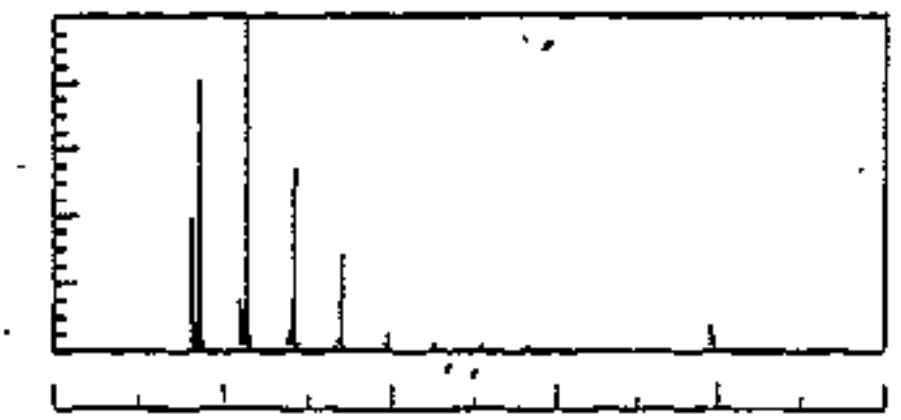

NIST Library Mass Spectrun Mareht



AMII.

Figure 3-9. HSGC-MS Analysis of Diesel Constituents Showing Total Ion Chronatogram, Mass Spectrum and Library Match

component of the PCB sample. These initial results indicate both bardware and software are properly functioning for the HSGC-MS. These data formats are compatible with the archive and mapper systens. The datafiles will be transferred over hardwire link upon integration with the mapper subsystem.

In a separate experiment, $100 \mathrm{ng}$ sample of diesel range organics (DRO) were analyzed. The data shown in Figure 3-9 indicates a close match be- tween the measured mass spectrum and the library. The implication is that the HSGC-MS is operating under conditions which provide data that are comparable to conventional GCMS.

The tests indicated that the High Speed GC MS functions in a manner which allows effective deterrnination of sample constituents in less than 40 seconds. 


\subsubsection{Sammary High Speed GC-MS Analysis}

The Phase II objective was to develop the necessary hardware and procedures for high speed GC. MS analysis of the toxic organics, using analog compounds similar to PCBs. The goal was GC separation and mass spectrometer identification in one minute to regulatory limits. The high speed GC-MS sensor is expected to accomplish GC separation, MS identification, MS library database search for identification and quantitation in less than 60 seconds. This capability will satisfy the Phase II success criteria of sampling analysis and data processing for GCMMS. Coupled with the Multisensor probe for automated sample extraction, preconcentration and transport this subsystem has established a new benchmark for speed and performance in organic analysis.

\subsection{Radfonuclide Sensors}

For the majority of radioisotopes of DOE interest two detectors were determined to best meet the functional needs. This was because most isotopes were atpha emitters ( $\mathrm{Am}, \mathrm{Th}, \mathrm{Pu}, \mathrm{U}$ ), and one was a beta enitter (Cs). The 3D-ICAS radionuclide sensor system includes both an Eberline diffused junction silicon detector for alpha emitting isotopes and a Eberline sealed gas proportional detector for beta/gamma emitting isotopes. A multichannel analyzer is integrated with the diffused junction detector for discrimination of the alpha isotopes. This design ensures that all the isotopes of interest will be identified.

The combination of the two detectors will ensure that the DOE specified types of radioactivity will be detected. The specific alpha emitring isotopes U-238, U-235, Pu-239, Pu-242, Am-241 and Th-230 are able to be identified and quantified. Totally beta/ganma activity will be reported as $\mathrm{Tc}-99$. Both Tc-99 and Cs-137 may be present as beta emit- ters, but $C s$ is not as important(toxic) as Tc. Although not done in Phase II, the gamma activity can be discriminated from the beta activity by simple mechanjcal shuttling of metal plate discriminators. A somewhat more complex, variable thickness shutter may be ased to provide beta energy level discrimination.

\subsubsection{Alpha Detection}

The starting point for the alpha detection was Eberine's existing commercial, product, the Alpha6. This device is typically used in air monitoring application for discrimination of low levels of air bome alpha isotopes. The alpha isotopes are discriminated on the basis of pulse height using a multichannel analyzer detennined from the penetration depth ('track') of the particle through the silicon substrate. The data are recorded as a function of energy in the multichannel analyzer. The discrimination is based upon "regions of interest" which are collection of channeis summed together to give a response for a particular isotope. A 1 inch detector was integrated into the MSP. The associated electronics were integrated into the control software for the sensors.

\subsubsection{Beta/Gamma Detection}

The starting point for the beta/gamma technology was Eberline's Gas Proportional detector and modular detector board used in various commercial products. The gas (argon-carbon dioxide mixture) undergoes ionization by incident radiation, a charge is coliected on the anode which is capacitively coupled to a comparator. Thresholds in the comparator discriminate noise and high energy alpha emission from the beta/gamma response. If gamma radiation is expected a $1 / 16$ " plate can be placed in front of the nube to discriminate it from the bera. A 2 inch diameter gas proportional tube was integrated into the Multisensor probe. The associated electronics were integrated into the control software for the sensors. 
3.2.3 Experimental Resilts

Shown in Figure 3-10 is an example of a detector sensitivity testing. The sample is thorium 230 embedded into concrete at $\mathrm{Bq} / \mathrm{cm} 2$ level (the regulatory level). The data show that within 60 seconds on a medium rough concrete surface sufficient data can be collected to identify the thorium 230 . In an-



Figure 3-10. Example of Data Measured from $1 \mathrm{~B} q / \mathrm{cm}^{2}$ of Thordum-230 Embedded on Concrete Surface

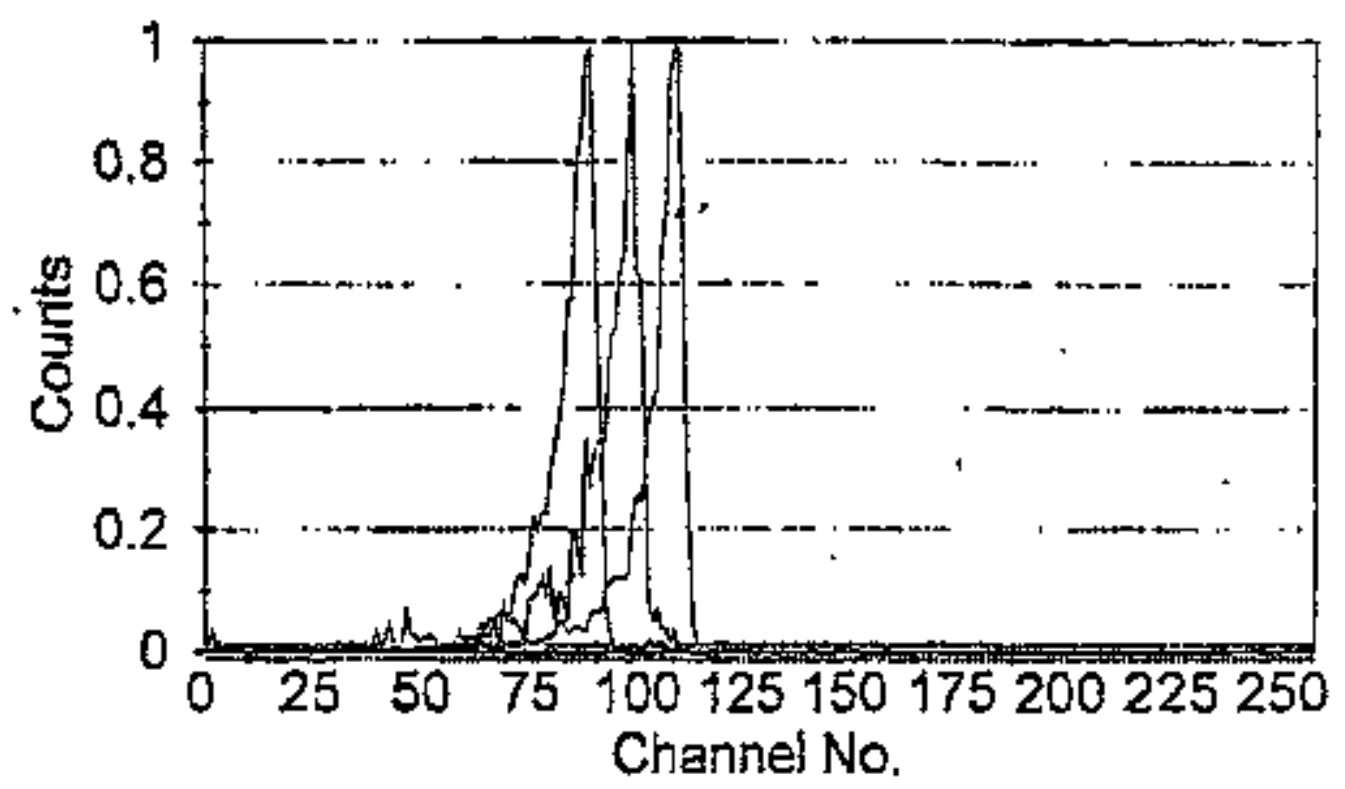

Figure 3-11. Example of Resolution for Discriminating Thorium, Plutonium and Americtum using Standard Samples 
other test standard samples of thorium, piutonium and americium were analyzed. Shown in Figuze 311 , is the data from this experiment. It is clear that stufficient resolution exists to differentiate these isotopes. No experiments were conducted on mixtures of these isotopes on concrete surfaces due to the difficulty of obtaining long term storage for the samples once the testing was completed. Based on the minor changes in the thorium standard and concrete samples it is expected that this sensor system will be capable of discriminating mixture of these isotopes on concrete.

Shown in Figure $3-12$ is an example of the alpha spectrum reconded during a simultaneous measurement of GC/MS and radionuclide data. For this tion health industries. The source was $0.5 \mathrm{~mm}$ from the detector and the measurement time was $30 \mathrm{sec}-$ onds. The data presented in Figures 3-6, 3-7, 3-8 and 3-12 indicate that the sensors system can make simultaneous measurements in near-real time.

The seasitivity of the gas proportional tube for beta and gamma detection was determined using $S$ tifferent isotopes of widely varying activity (dpm). The data shown in Table 3-1 indicates an acceptable level of sensitivity for several beta emitting isotopes as measured in the background activity of the Eberline facility in New Mexico.

The final column of data is the 10 second minimum detected activity (MDA) at the 95\% con-



Figure 3-12. Example of Therinm-230 Measured Simultaneously with the ESGC-MS Data

analytical tun the sample was a mixture of PCBs and Thorium - 230 alpha standard. The cortesponding GCMS data is shown in Figores 3-6, 3-7 and 3-8 and the results for the PCB sample are described in Section 3-1. The peak of the alpha spectrum occurs at the energy level of $4.7 \mathrm{MeV}$ indicating it is thonium 230. The alpha source is a sample of thorimm embedded on a nickel substrate. These source are typically used as check standards through the radia- fidence level for the different isotopes. This data indicate that the gas proportional tube is an effective detector for beta activity from surface as measured in air for sbort 10 second analysis times.

There results indicate that the radionuclide detectors have the necessary sensitivity and discrimsination ability to detect the radionuclide of DOE interest. 
Table 3-1. Indicates an Acceptable Level of Sensitivity for Several Beta Emitting Isotopes

\begin{tabular}{|c|r|r|r|r|}
\hline Isotope & Activity (dpm) & Half Life (Yrs.) & $\begin{array}{c}\text { Current } \\
\text { Activity }\end{array}$ & $\begin{array}{c}\text { Gross Count } \\
\text { Rate }\end{array}$ \\
\hline SrY-90 & 6790 & 28.6 & $513 !$ & 1080 \\
\hline Ba-133 & 1410000 & 10.5 & 1311427 & 47300 \\
\hline Tc-99 & 16320 & 213000 & 16320 & 4800 \\
\hline Pm-147 & 19296 & 2.6234 & 8435 & 977 \\
\hline C-14 & 4099.2 & 5730 & 4097 & 328 \\
\hline
\end{tabular}

\subsection{The Molecular Vtbrational (MVS) Sensor}

The sensor for identification of base materials of interest to DOE (concrete, transite, asbestos, wood, and other organics) is a combination of an IR reflection sensor and a Raman scattering sensor.

During Phase II it has been demonstrated that the MVS probe should be based prinarily on an extended near-infrared (ENIR) spectrometer equipped with a heavy metal finotide (HMF) fibet-optic probe and secondarily on a 785-nm Raman spectrometer equipped with a silica fiber-optic probe. High quality spectra bave been obtained from most materials investigated within 1 minute for both probes.

In an extensive test of a mumber of Ranan spectrometers and laser wavelengths, it was shows that excitation at a wavelength of $785 \mathrm{~nm}$ gave the optimal combination of fluorescence rejection, minimal sample heating, portability and low data acquisition times to achieve a given signal-to-noise ratio
(SNR). The Raman probe arrangement has been modified from the type studied in Phase $\mathbf{I}^{\mathbf{1}}$, in which the probe-hear investigated incorporated 6 collection fibers around one excitation fiber (the so-called 6-around-1 arrangement) with no optical filtering. The optimum probe configuration was found to be a single input fiber with a notch filter motinted at the end so that no Raman scattered radiation from the silica ffber reached the sample. The radiation seattered from the sample is then passed through a second optical filter to remove the Rayleigh-scattered light (at $785 \mathrm{~nm}$ ) and pass all the longer-waveleagth (Raman scattered) radiation which is then focused into a single ootput fiber. The second filter prevents the Rayleigh-scattered radiation from giving rise to Raman scattering in the output fiber. A commercial spectrometer and probe head manufactured by Kaiser Optical \$ystems, Inc. were identified as being optimal for the measurement of the Raman spectra of solid samples with a low Raman cross-section (such as asbestos). This instrument is now in operaton at the UI and will be incorporated into the Phase III mistisensor probe. 
Two types of software for classification and/ or identification of the samples have been studied. In the fist, commonly known as spectral searching, the measured spectrum is compared directly to a library of reference spectra and the absolute difference between these spextra is calculated and summed over all wavelengths. The reference spectrum yielding the smallest sum is the best match to the spectrum of the unknown. This approach proves to be most useful when the spectrum of the unkrown contains several narrow bands that are easily distinguished from the spectral baseline. In this case, an automated baseline comection routine that has been developed at UI can be applied prior to the application of the spectral searching program. For many of the samples, however, the spectra are not of the ideal form for spectral searching. For such samples, other approaches must be appiied. Two such techniques were investigated: a relatively well-known algorithm known as principal components analysis (PCA), and a new form of spectral classification inwolving the use of self-organized mapping (SOM) neural networks. The latter approach has proved to be more robust than etther spectral searching or PCA and will form the basis of the classification software in the final field version of the MVS probe.

The results obtained with the silica fiber-optic probe analysis of the materials of DOE interest indicated that NIR diffuse reflectance (DR) spectrometry with a silica fiber-optic probe can be used to analyze all materials except those that totally absorb NIR radiation. It was also shown that NIR DR spectrometry could be used to distinguish between the five major types of asbestos. However, the spectral features that are of importance for distinguishing asbestos from analogous sampies such as bricks and concretes are so weak that they can be lost in the baseline noise if the corresponding measurements are made using detectors operating at temperatures appropriate for freld measurements. For such samples, access to the much stronger fundamental
$\mathrm{C}-\mathrm{H}$ and $\mathrm{O} \cdot \mathrm{H}$ scretching modes would be highly beneficial. For this reason, we investigated the feasibility of extending the wavelength range of fiber-optic DR measurements down to about $2500 \mathrm{~cm}^{-1}$.

Experimentally, we have shown that, for samples for which ENIR DR spectra do not contain enough useful information to permit rapid identifncation, Raman spectrometry often does.

Thus ENIR DR and Raman spectrometry often prove to be highly complementary techniques.

\subsubsection{Extended Near Infrared Spectrometry}

An experimental fiber-optic probe for DR measurements using heavy metal fluoride fibers and incorporating a thermoelectrically-cooled mercary cadmium telluride detector was purchased from Galileo Electro-Optics Corp. and interfaced to a ATI Mattson Genesis Fourjer transform spectrometer configured to cover the spectral region between 1 and $4 \mu \mathrm{m}$. The combination of this spectrometer and the heavy metal fiber DR probe enabled high quality spectra to be measured when the probe head was in direct contact with the sample. Typical spectra measured in a time of 45 seconds with this instrument are shown in Figure 3-13.

For a fieldable ENIR probe, direct surface contact is undesirable because of possible probe contamination. Therefore a second probe design was tested which employed a lens to focus on surfaces spaced a 3 mon distance. With this design there is a small change in relative band intensities in the observed DR spectra. This effect can be accommodated by including approptiate reference spectra in the database for spectral identification or through a software correction. The approach for this compensation will be determined in the remainder of Phase II. 




Figure 3-13. ENIR DR Spectra of Actinolite, Concrete, Wood and Polystyrene Fosme Measured in the Contact Mode

\subsubsection{Raman Spectrometry}

Most CCD-Raman spectra were acquired on a Renishaw Raman spectrometer utilizing a microscope as the sarapling device. The 785 -nm diode laser provided a maximum power of $2 \mathrm{~mW}$ at the sarnple. Excitation with gas lasers emitting at 632.8 and $514.5 \mathrm{~nm}$ was also tested but fluorescence overwheimed the Raman spectrom features for most materisls of interest. Different lenses allowed standoff distances between 1 and $10 \mathrm{~mm}$ to be tested. Singleand multiple-scan accumulation spectra were collected at approximately $6-\mathrm{cm}^{-1}$ resolution asing $\mathrm{CCD}$ detector charge integration times from 1 to $120 \mathrm{sec}$ onds. Band positions are accurate to within $2 \mathrm{~cm}^{-1}$. The results obtained with this spectrometer without the fiber-optic probe were sufficiently encouraging that we investigated other commercially available
Raman spectrometers to which fiber-optic probes had been interfaced. Of these instruments, the one yielding spectra of solid sampies with by far the highest SNR was the Kaiser Optical Holoprobe Raman spectrometer.

The Kaiser Holoprobe is a state-of-the art NIR Raman spectrometer optimized for use with 785-nm excitation. The system comprises a 50-nW external-cavity-stabilized diode laser providing a maximum laser power at the sample of $20 \mathrm{~mW}$, a transtnission grating, a CCD detector, and a stateof-the-art fiber-optic probe packaged in a smaller volume than the Renishaw spectrometer. This system offered a useable Raman shift wavenumber range of $50-3500 \mathrm{~cm}-1$ with $785-\mathrm{nm}$ excitation. 


\subsubsection{Tests for the Appropriate Excitation Wavelength}

When the 632.8-nm and the \$14.5-non laser lines were used for excitation, the spectra are so severely affected by molecular and/or atomic luminescence that no Raman bands can be observed above the background. Thus the wavelength for the excitation laser for the MVS Raman probe had to be in the near infrared; in practice the only two wavelengths between which a choice bad to be made were 1064 and $785 \mathrm{~nm}$ (see Table 3-2). The $785 \mathrm{~nm}$ excitation was selected because of the greater strength of the Raman effect and shorter sample time required at that wavelength.
The filtered probe-head arrangement prevents any Raman scattering from the fiber-optic cables from obscuring the Raman signal from the sample. The Kaiser Optical System ${ }^{3}$ Raman probe, bas been identified as optinal for the 3D-ICAS application based on the signal throughput of the fiber-probe, the low Raman cross-sections of some of the samples of interest in this project (especially, asbestos and concrete), the wavenumber range $(\Delta v=50-1000$ $\mathrm{cm}^{-1}$ ) needed to identify DOE materials of interest.

A recent study sponsored by the DOE at Westinghouse-Hanford ${ }^{4}$ has shown for non-reflec* tove non-fluorescent samples that a 6-around-1 probehead with a flat-tip termination can yield up to an

Table 3-2. Wavelength for the Excitation Laser for the MVS Raman

\begin{tabular}{|c|c|c|c|c|c|c|c|c|}
\hline \multirow[t]{2}{*}{ Material } & \multicolumn{4}{|c|}{$\begin{array}{l}\text { Intersianpte } \\
\text { Variability }\end{array}$} & \multicolumn{4}{|c|}{$\begin{array}{c}\text { Siganl-to-Noise Ratio } \\
\text { (degraded by fluorescence and } \\
\text { sample heating) }\end{array}$} \\
\hline & 514.5 & 632.8 & 785 & 1064 & 514.5 & 632.8 & 785 & 1064 \\
\hline Concrete & - & I & 4 & 1 & $=$ & VP & $\mathbf{M}$ & vp \\
\hline Bricks & - & I & 1 & 3 & - & VP & VG & $G$ \\
\hline Asbestos & 1 & 1 & 3 & 3 & vP & VP & $G$ & $M-P$ \\
\hline Transite & + & 1 & 1 & 3 & - & $\mathbf{P}$ & $\mathbf{P}$ & M-P \\
\hline Porcelain & 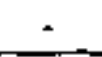 & $\therefore$ & 1 & 2 & $=$ & $\therefore$ & VG & G \\
\hline Woods & 1 & \pm & 1 & 1 & VP & $\mathbf{P}$ & $\mathrm{P}$ & VG \\
\hline Asphalt & 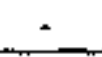 & 1 & 1 & 1 & - & vp & VP & vP \\
\hline Polymers & 4 & 3 & 2 & 2 & $\mathrm{G}-\mathrm{M}$ & G & G & $\mathbf{G}$ \\
\hline Organics & . & 3 & ר & & & & $\mathbf{G}$ & G \\
\hline
\end{tabular}

\subsubsection{Probe-head Arrangements}

The fiber-optic probe design for Raman spectrometry has changed significantly from the 6around-1 arrangement tested in Phase I to the filtered probe-head now used. order of magnitude more signal than the filtered probe-head arrangement. It should be noted, however, that the filtered probe that was used for this study had only a $10 \%$ throughput. The probe head selected by UI for the 3D-ICAS has a throughput of approximately $50 \%$; thus, all else being equal, there is only factor of two difference between using the Kaiser probe and the Westinghouse tested design. 
The reduced silica fiber scattering backgrousd for highty reflective materials more than compensates for this smatl reduction in throughput efficiency.

It has been shown both at the $\mathrm{UI}$ and in other DOE funded efforts ${ }^{56}$ that if the background radiation caused by the Raman spectrim of silica in the input fiber is not filtered out the Raman signal from the sample can be significantly degraded or completely obscured. In Figure 3-14, the Raman spec- bands are observed between 200 and $1000 \mathrm{~cm}^{-1}$. (It should be noted that Balsa wood is not a particularly weak Raman scatterer.)

In Figure 3-15 the Raman spectra of two asbestos minerais, chrysotile and crocidolite, are presented as well as the Raman silica background from the 2 meter 6-around-I Raman probe. It can be seen that the silica background will obscure the strong vibrational bands of crocidolite (Figure 3-15b) and



Ramsa Shift (ER-1)

Figure 3-14. Typical Raman Spectroscopic Performance of an Unnitered f'ocased Fiber-Optic Probe

trum of balsa wood measured through a 2-meter unfiltered 6-around-1 probe-head is presented. A comparison of the resultant balsa spectrum (Figure 314c) produced by subtraction of the silica background from the original probe-recorded spectrum of balsa with the balsa spectrum recorded in the macronchamber of the spectrometer (Figure 3-14d) shows that the SNR bas been significantly degraded when onfiltered fiber-optic probe-heads are used. This is especially obvious in the wavenumber range below $1000 \mathrm{~cm}-1$ where spurious intensities for vibrational that the chrysotile bands may also be lost in the background. The asbestos minerals are very weak Raman scatterers (at least one order of magnitude weaker than the sample of balsa wood shows in Figure 314). Thus even though spectra measured with the 6 around-1 fiber-probe have not been obtained for the asbestos minerals it appears clear that the noise as sociated with silica background which is left in the resultant spectrum after background suberaction will totally obscure any asbestos signal from being observed. 


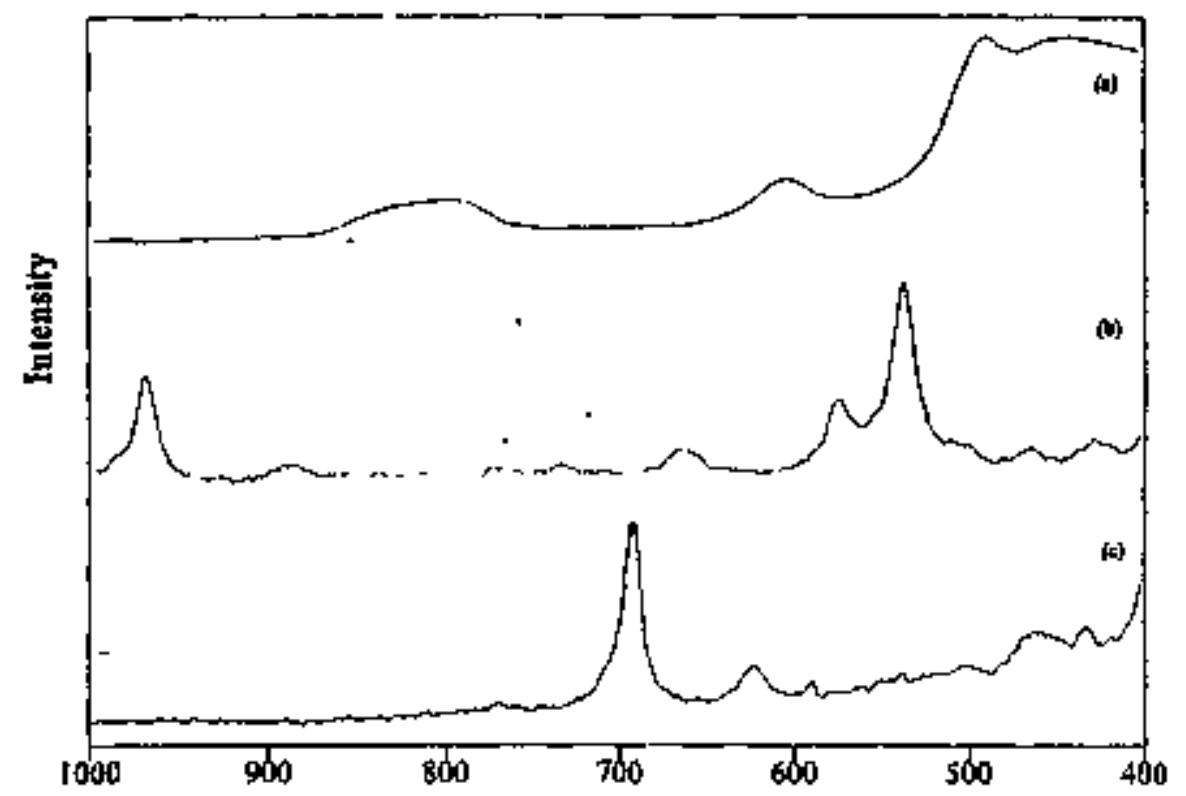

Raman Shift (em-1)

Figure 3-15. Raman Spectra in the Wavenumber Kange

In contrast, the filtered probe-head design of ser power; the signal level of these spectra is suffiKaiser has been used to obtain Raman spectra of cro- cient to allow the mineral to be identified, as shown cidolite within two minutes using only $5 \mathrm{~mW}$ of $12-$ in Figures 3-16b and 3-16c. All the intense feabures



\section{Rasian Stift (cm-1)}

Figure 3-16. Raman Spectra of the Asbestos Mineral Crocidolite Recorded 
in the reference spectrum (Figure 3-16, measured in about an hour) can be seen with a SNR of greater than 3 in spectra measured in 2 minutes with the Kaiser fiber-optic probe.

The schematic of the Kaiser bolographic probe (Figure 3-17) shows that the probe-inead comprises (i) a laser bandpass filter incorporated in the probe-head case after the radiation has emerged form the illumination fiber and (ii) holographic Rayleigh possess crucial vibrational information in the region $800-50 \mathrm{~cm}-1$, the chisel-ip probe approach pioneered at Dow Chemicals9 and the flat-tip approach5 proved to be unsuitable for the DOE's specific needs. They have, therefore, not been adopted becalnse both methods fail to meet the sample-dictated criteria necessary for routine analysis. The results of Phase II have shown that the Kaiser Holoprobe Raman fiber-optic probe is the best available design for studies of weakly Raman scattering solid materials, especially

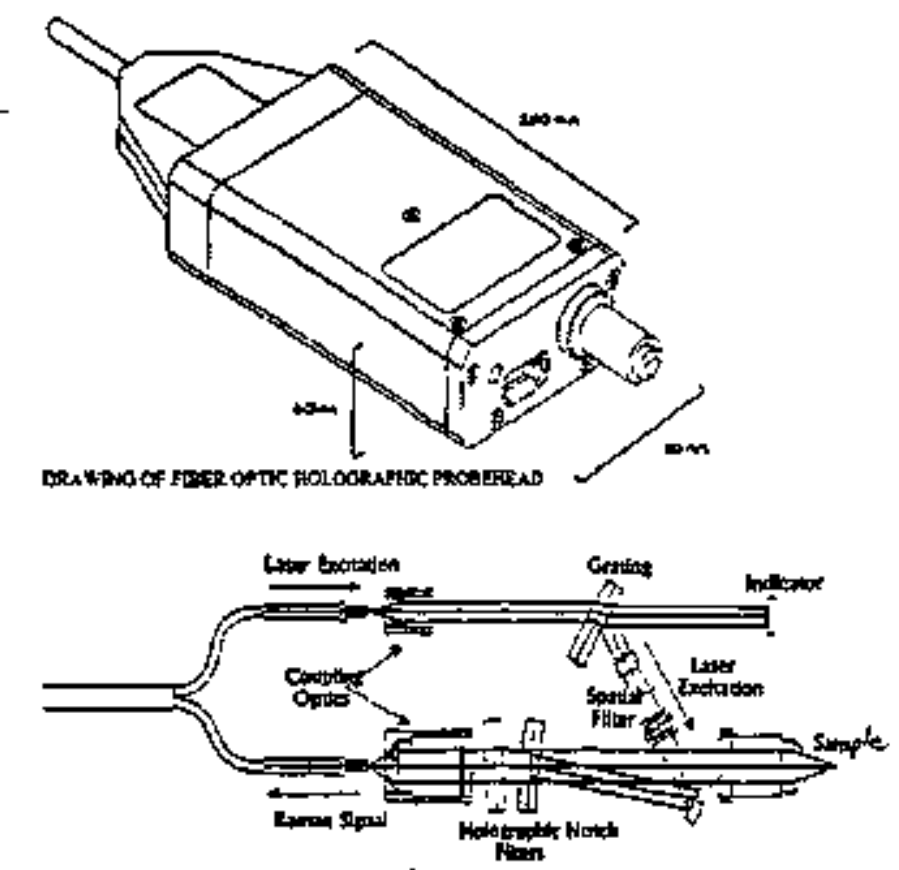

Figure 3-17. Diagrammatic Representation of the Kaiser Holographic Probe-Head

notch filters which prevent refiected laser radiation from entering the collection fiber. These two conponents satisfy the silica background rejection requirement demonstrated above. A third important component is an emission indicator built into the probe head. This indicator should allow an untrained operator of the Raman spectroscopic probe to verify if the laser operation on a regular basis without the need for expensive test equipinent or extensive sensor down-time.

In conclusion since a number of materials of interest in this project (most importantly asbestos) when the wavenumber range below $800 \mathrm{~cm}-1$ is important (as it is for asbestos).

\subsubsection{Software for Identification and Classification}

Two types of software for classification and of identification of the samples bave been studied, spectral searcting and self-organized mapping (SOM) neural networks. In spectral searching, the measured spectrum is compared directiy to a libray of reference spectra; the absolute difference between the spectrum of the unknown and each reference 
spectrum is calculated at each wavelength and summed over all wavelengths. The reference spectrum yielding the smaliest sum is the best match to the spectrum of the unknown. Spectral searching proves to be most useful when the spectrum of the unknown contains several narrow bands that are easily distinguished from the spectral baseline. In this case, an automated baseline correction routine that has been developed at UI can be applied prior to the application of the spectral searching program.

Unfortunately, the spectra of many of the samples encountered in this project ane not of the ideal form for spectral searching. For such samples two other techniques were investigated: principal components analysis (PCA), and self-organized mapping (SOM) neural networks. For both sech- two-dimensional plot is produced in which it is hoped that samples of a given type wiil form a cluster in a given region of the plot. In PCA, for example, the ordinates of the plot are the scores of the first two principal components ( $\mathrm{PC} 1$ and $\mathrm{PC} 2$ ). When the ENIR spectra of a number of samples of brick, cortcrete, asbestos and polymers are subjected to the PCA, quite poor clustering results (Figure 3-18). With the use of SOM neural aetworks, on the other hand, most samples of a given type fell within weildefined regions of the map (Figure 3-19). Neural computing with self-organizing mapping has proved to be more robust than either spectral searching or PCA and will form the basis of the classification software in the final field version of the MVS probe, using both Raman and ENIR reflectance data

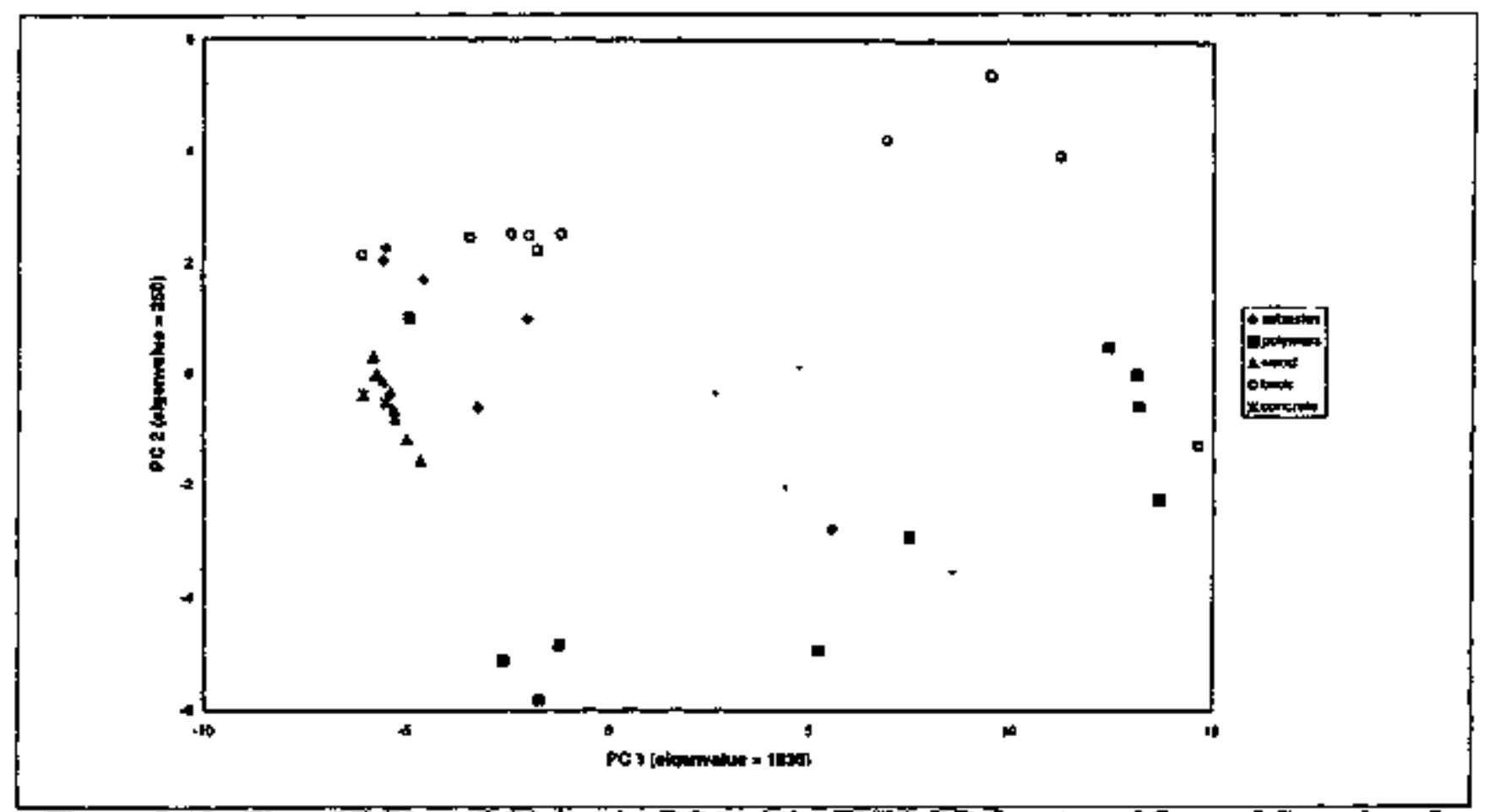

\section{Figure 3-18. Plot of PC1 vs. PC2 tor the PCA of the ENIR DR Spectra of Several Samples of Asbestos, Polymers, Wood, Brick and Concrete}

niques, the dimensionality of the input data is reduced from a fairly large number (the number of data points in each spectrum, often several hundred) to a much smailer number (usually two). In each case, a

\subsection{D-ICAS System Integration}

The 3D-ICAS system uses its coherent laser radar (CLR) to obtain 3D facility maps, then uses 


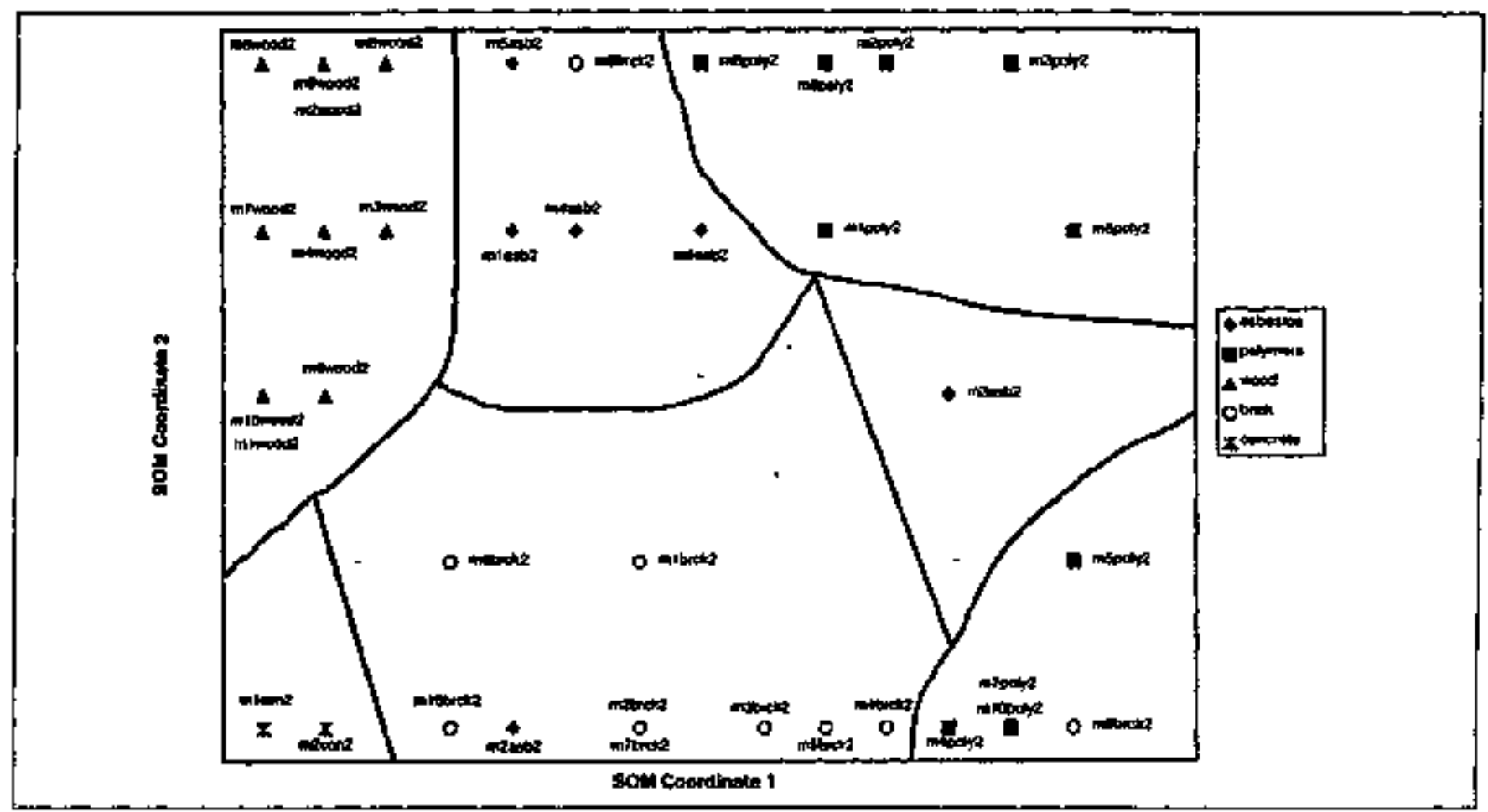

Figure 3-19. Plot of Coordinate 1 vs. Coordinate 2 for a SOM

the CIR to guide a robot arm borme multisensor probe along surfaces for contamination detection, 3D mapping and archiving. A substantial portion of the development effort has been devoted to obtaining the integrated functionality of these subsystems. We will describe some of these subsystems and their interaction briefly. We will then provide performance examples of the 3D-ICAS "eyes" the CLR 3D Mapper.

\subsubsection{Robot Arm}

Robot arm control and manjpulation is an important part of the Phase II work. Integration of control of the robot arm shows advancement towards a fiejdabie system. During Phase II a model 465A robot arm from CRS Robotics (CRS) was selected, programmed and used to manipulate the sensor probe in a limited area.

The CRS 465A robot arm has about the same reach as a human's arm with more flexibility. The arm is not operated at full speed. The CRS C500 controller is programmed in the Robot Applied Programming Language, RAPL II, a language developed by CRS which is a derivative of BASIC and C. In Phase II the robot arm will be attached to a mobile platform allowing for foll working space access.

\subsubsection{Multisensor Probe}

The Phase II multisensor probe comprises the GC sensor head, two radionuctide sensors, one for alpha particles and one for beta/gamma emissions, and four proximity sensors. Three tetrabedron tracking targets are mounted on the outside of the probe. The probe is cube $6 \times 6 \times 6$ inches and weighs 6 lbs.

The Phase III probe will be essentially the same with three modifications. First, several tracking targets will be added to provide compiete tracking capability. Second, the MVS sensor will be in- 
tegrated with the other sensors on the probe. Third, proximity sensors will be selected and mounted in sach a way that curved surfaces can be handled.

\subsubsection{Proximity Sensor}

The Phase II system used commercial proximity sensors mounted at the four corners of the face of the probe. These proximity sensors provide a capability to move the probe up to a flar surface and maintain a standofi distance of $1 \mathrm{~mm}$ or more.

\subsubsection{Robot Arm Patform}

The Phase II system was not required to pro. vide mobility for either the mapper or the robot arm. Portability was provided by mounting eack unit on a wheeled tripod and moving them manually. For the Phase III demonstration the 3D-ICAS will take advantage of DOE developed mobility platforms for both the 3D mapper and the sensor robot arm.

\subsubsection{Control Strategy}

The multisensor probe moves to each point specified in the survey list in the order in which they appear in the list generated by the integrated workstation. Movement between points is along a standard sequence of steps. First, the muitisensor probe moves from the rest position along a straight line to a position near the next survey point, sotating along the way so that the face of the probe is nearly parallel to the surface at the end of the segnent. Second, the probe moves toward the surface until the proximity sensors detect the surface. The final approach is accomplished in small steps under control of the proximity sensors. The sensor system is cornmanded to acquire the contaminant sample and initiate processing. Then the probe moves along a straight line to the arm's rest position.

When the probe has settled at each point, the CLR tracker is activated and a scan of the tracking target is made. The scan data are processed to pro- vide an accurate estimate of the probe's location and orientation.

The Phase II probe controi strategy moves the probe up the desired spot and atl contaminant sensing is done with the probe in one place. The surface being sampled was assumed in Phase II to be flat over the entire face of the probe so that moving the probe up to the surface with the face of the probe parallel to the surface was an acceptable approach strategy. In Phase III a more robust approach strategy will be implemented. First, one will be able to move the probe up to a single spot, or move each of the four sensor heads in sequence to the same spot. This will allow the probe to make contaminant measurements at the same spot. It also permits the system to survey curred surfaces such as pipes. Finally, with the sampling sequenced properly, i.e. with the MVS and RN sampies taken first, the GC sampling can be suppressed if the MVS indicates that organic contaminant leveis are so high that the GC sensor could be contaminated. In this case the high sensitivity of the GC-MS is not needed.

\subsubsection{End Effector Tracking Function}

CRC has developed an efficient approach for the CLR for tracking location and orientation of a robot end effector. Analysis, simulation, and laboratory experiments demonstrate the effectiveness of this technique. The tracking system has been integrated with the 3D-ICAS Phase II system demonstration. The tracker provides 6-DOF (X, Y, Z location and Yaw, Pitch, Roll orientation) tracking using a symmetric tetrahedron target.

\subsubsection{Tracking Targets}

The tracking target is a symmetric tetrahedron consisting of an equilateral triangle base and three identical faces: Figure 3-20. Reflective paint is applied to the entire target except along the edges between faces. 


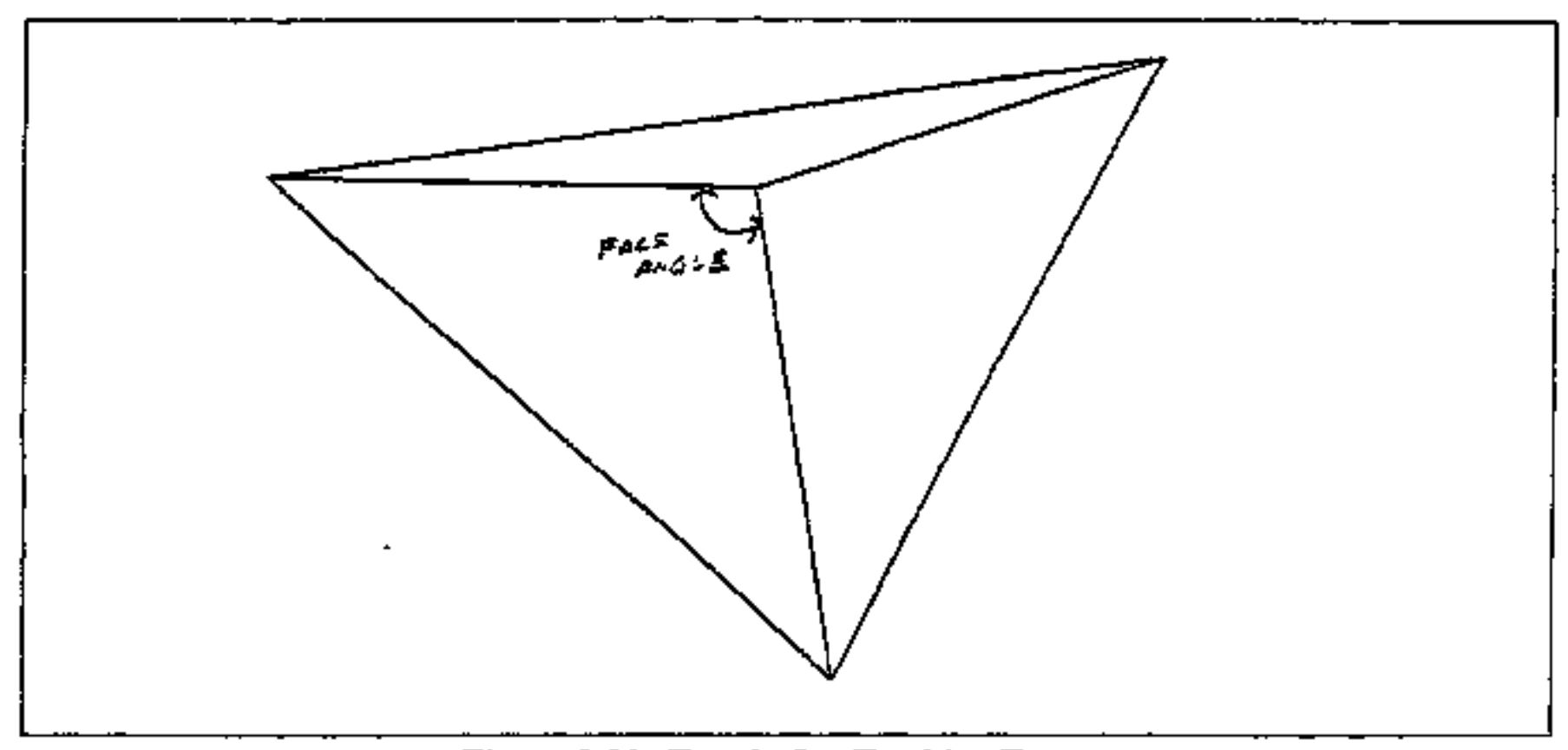

Figure 3-20. Tetrahedon Tracklng Target

Target size was constrained by the size of the probe and the face angle was selected considering location accuracy, orientation accuracy, and the number of targets required for full visibility. Targets with 110 degree face angle were specified for the Phase II demonstration.

\subsubsection{Position Estimation Algorithm}

The technique consists of scanning the CLR beam in a circular pattern around the apex of the target. The center and radius of the scan are preset so that the scan encircles the apex and remains on the target for the entire scan. Since the approximate location and orientation of the target are known, the desired scan pattern is easily determined. The CLR retums range, azimuth, elevation, and signal quality for each measurement point during the scan.

The first step of the position estimation algorithm is to estimate the three planes that best fit the three data sets independestly. The intersection of the three planes determines the iocation of the apex of the target. The vectors normal to each plane are immediately available. These vectors provide the target's orientation. The second step is to fit the independent normal vectors by a set of normal vectors constrained in such a way that they correspond to a tetrahedron target with the specified face angle. The location of the apex is recomputed after the best fit orientation is determined. This estimate of location and orientation will be used as the starting point for a steepest descent optimization in Phase III.

\subsection{Target Tracking Results}

Two targets were fabricated by a commercial machine shop. Target 1 was specified with a 90 degree face angle and target 2 with a 110 degree face angle. Tests of the targets showed that the angles between the faces ranged from 88.60 degrees to 91.31

degrees whereas they should have been 90.00 degrees. Corresponding angles for target 2 were much more consistent, showing a spread of 0.03 degrees, 
but they were over a degree different from the design value. Each target was evaluated by taking ten concentric scans with different madii, estimating the location of the apex and orientation, and computing the root-sum-squared standard deviations. Table 33 summarizes the results.

Table 3-3. Root-sum-squared Standard Deviation (rsssd) from Ten Scans

\begin{tabular}{|c|c|c|}
\hline & Target 1 & Target 2 \\
\hline $\begin{array}{c}\text { Design face } \\
\text { angige }\end{array}$ & 90 degrees & 110 degrees \\
\hline $\begin{array}{c}\text { Apex escinate } \\
\text { rsssd }\end{array}$ & $0.97 \mathrm{~mm}$ & $0.63 \mathrm{~mm}$ \\
\hline $\begin{array}{c}\text { Direction angle } \\
\text { Estimate rsssd }\end{array}$ & 1.45 degrees & 1.0 degrees \\
\hline
\end{tabular}

The results indicate that the flatter target gives better results and estimate accuracy on the order of 0.6 millimeters and 1.0 degrees should be possible.

\subsubsection{Database Function}

The database capability developed for the 3D-ICAS system serves as a repository for all of the data collected by the system. As the data are gathered, the system automatically sorts, keys and archives it for easy retrieval and display by the Integrated Workstation (TWOS). The data can also be made available for external clients as a server in an overall facility contamination management system.

Data are recorded for each survey point. The location of the sensor head is recorded precisely in facility coordinates along with the entire data structure from all of the sensors. The data structure includes raw data from the radionuclide $(R N)$ and High-Speed Gas Chromatograph/Mass Spectrograph (HSGCMS) sensors. Data from the Molecular Vibrakional Spectrometer (MVS) will be incorporated during Phase II in accordance with the three phase development schedole. The data structure also includes processed assignments for four organic compounds provided by the HSGC/MS. Finally the database holds any specific images generated from the raw 3D mapper đata.

International Business Machine's (IBM) industrial database DB2 was selected for use in the 3D-ICAS system after surveying the leading largescale commercial database vendors.

A prixary consideration in the selection was the ability to directly support structured query language (SQL) in multi-platforms. SQL is used in the 3D-ICAS access and storage of the data. This alIows future expansion of the system to provide the database information to other users in a networked environment. DB2 also runs on a wide variety of computers including workstations, PCS, and mainframes.

In 3D-ICAS DB2 will be fun on the laser radar control computer. Using the preemptive raultitasking capabilities of $O S / 2$, the database will run in a separate session to ensure data integrity and responsiveness. This configuration will be cartied over to Phase III.

\subsubsection{Data Archiving and Retrieval}

The database is setup as a set of tables to store the following information (see Table 3-4):

Queries can be made on all of the fields. The defined primary key fields used for sorting are the Facility Identification and Date, although any data field may be selected and sorted.

Raw data from the Molecular Vibrational Spectrometer will be integrated into the database function during Phase III. 
Identification

- Facility Identification (Agency, Plant, Building, Room)

- Date, time

Mapping

-

Location of fiducial marks in room coordinate frame

- 3D mapper data in room coordinates

- Any generated facility scenes

- Bounding box of coordinates for each 3D mapping run

\section{HSGC/MS}

-

3D coordinates of each sample point (in room coordinates)

- Gas chromatogram for each point

- Mass spectrogram for each peak in each gas chromatogram

- Mass of each of five compounds at each point (PCB, fivel, volatile organ ics, explosives, other)

RN

- $\quad 3 D$ coordinates of each sample point (in room coordinates)

- Alpha spectrum

- Beta-Gamma count

- Activity level of each of six isotopes (U235, U238, Pu239, Pu242, Am241. Th230)

\subsubsection{Data Display: Facility Scene}

A facility scene image is getzerated from the 3D facility frame mapper data by selecting the area to display and an optical viewpoint and converting range from the viewpoint into a rendered image. The facility scene is rendered in black and white so that subsequent contamination overlays in color will be highlighted.

\subsubsection{Data Display : Contaminants}

When a facility scene is displayed on the IWOS, the database can be queried for related survey information. The data can be overlaid on the facility scene as scarter contour plots or displayed in one of several different graphical or list windows.

Contaminant concentration levels are presented as thresholded scatter diagrams rather than continuous line contour plots because the interpolation ingerent in constructing contintous contours could produce an erroneous picture of the contamination, especially when the survey points are widely separated. Each contarninant is displayed in its own window. The window contains the gray scale facility scene overlayed with contamination levels in color. An asterisk is placed at each location where a measurement was made. The color of the asterisk depends on the concentration of the contaminant. Green, yellow, red, and blue indicate no, light, medium, and heavy contamination respectively. Default thresholds for defining the four contamination levels are provided; the user may change the thresholds.

More detailed displays of contamination data are keyed from the facility scenes with contamination overlays on them. For any contamination display, the operator can select a location to get detailed information about with the mouse. Radionuclide activity levels are displayed in tabular form. Gas chromatograms are displayed in graphical form. Masses of the five organic compounds are displayed as a bar graph. Secondary displays consisting of mass spectrograms are obtained by mousing on desired peaks in the gas chromatogram; mass spectrograms are disglayed as graphs and also in tabular form. An example of these displays is shown in Figure 3-21. Displays of data from the Molecular Vibrational Spectrometer will be integrated into the display function during Phase III. 


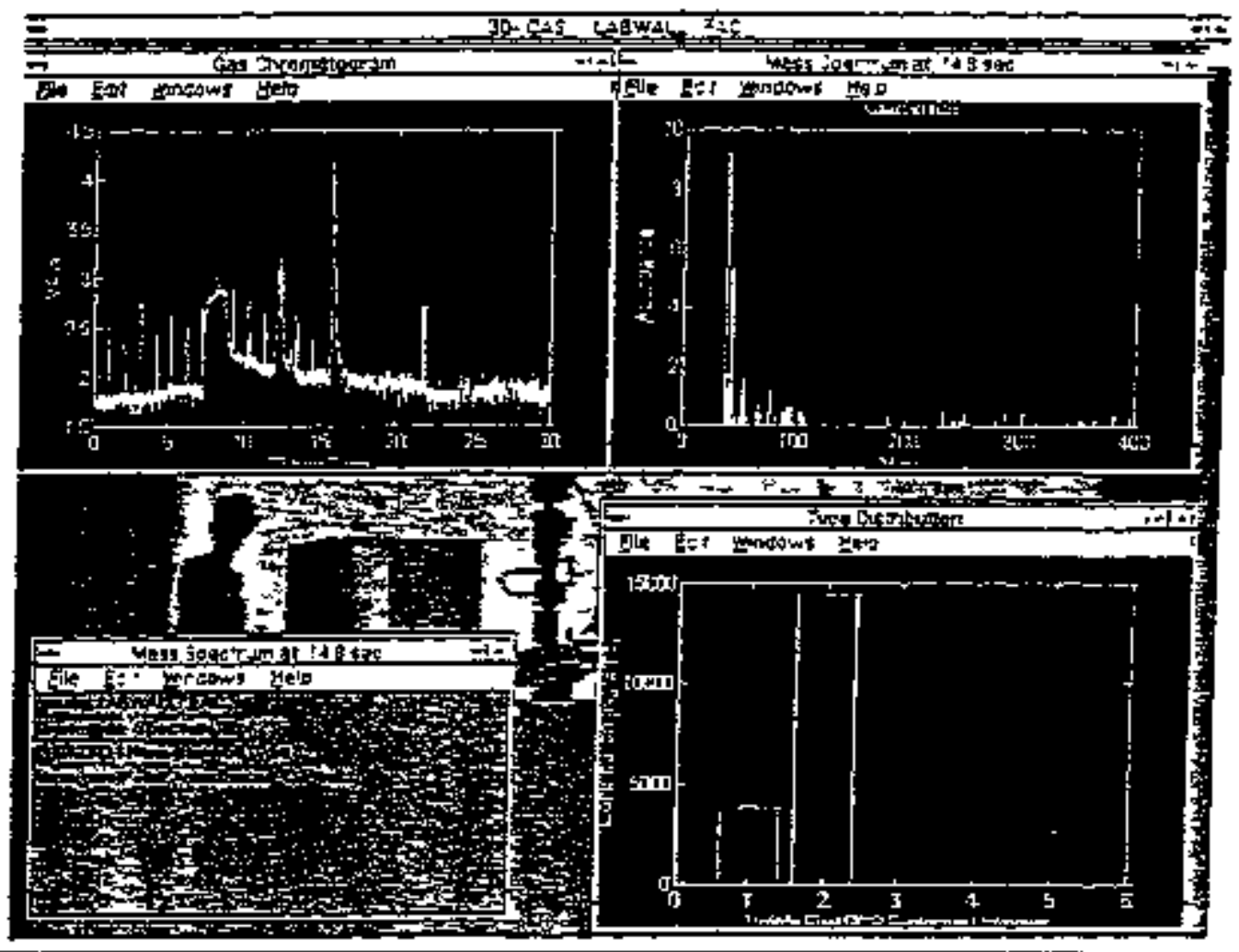

Figure 3-21. Facitity Seene Contaminant Data Display

\section{The CLR 3D Mapper}

The CLR 3D Mapper, which serves as the robotic eyes for 3D-ICAS, has continued to be refined with other program support during the 3DICAS Pbase II development effort. A CLR 3D Map. per to be dedicated to 3D-ICAS is being completed during Phase 17 . The accuracy of the CLR 3D Mapper is critical to the development of reliable facility maps and for accurate robotic sensor end effector trackitg and gujdance. An example of the mapping accuracy of the CLR 3D Mapper is shown in Figure 3-22, which shows a 3D image of a dime taker at four meters range.

\subsection{Application and Benefits}

The completed 3D-ICAS will be directly applicable as a system to DOE facility characteriza- tion decontanination and decommissioning. The sensor subsystems of 3D-ICAS represent an advance in portable real-tine chemical analysis for chemical constituents and dangerous materials of interest to DOE. The CLR 3D mapping stibsystern, developed outside of the 3D-ICAS program, can perfom as a critical subsystem on a wide range of DOE robotic applications and $\mathrm{I}$ industrial metrology applications.

Benefits of 3D-ICAS High Speed Contarni. nantBase Material Analysis for Overall Decontamination and Decommissioning Operations

3D-ICAS site chemical analysis is expected to improve the technological capabilities of current chemucal analysis methods by combining state-ofthe-art sample preparation techniques, High Speed GC, time-of-flight mass spectrometry (TOF-MS), molecular vibratıonal spectrometry, and radionuclide 




Figure 3-22. 3D Image of a Dime taken at Four Meters

analysis into a system that can be operated at a field site to provide real-time, reliable qualitative and quantitative results. The avatlability of this technology will positively impact DOE clean-up operations in the following ways:

- Improved Pefformance (faster, more accurate)

- Cost Reduction

- Reduction of Health Risks

- Reduction of Environmental Risk

- Improved Operations

- Reduced Time for Remediation

- Waste Minimization

- Supporting Overall Institutional Goals

Improved Performance: The quality of analytical data generated by 3D-ICAS chemical analy- sis instrumentation will equal or exceed that currently being generated by conventional Jaboratories. Sample automation and elimination of the extersive sample handling associated with off-site storage and analysis will improve the reliability of the data as well as significantly reduce sample preparation time. High speed GC techniques will produce chromatographic peaks more than 100 times sharper than conventional separations and improve the detector performance for a given mass of atalyte. High speed $G C$ will also reduce the separation time from $20-45$ min for a typical run down to t00 seconds or less, resulting in faster analysis times. The Radionuclide analysis may not provide more sensitivity than conventional laboratory analysis, but the direct real-time field results will certainly produce the required data expeditiously. Molecular vibrationai spectrometric analysis will identify inorganic and organic surface materials and moderate (down to low parts per mil- 
lion) levels of contaminations, at high spatial resolution, in saraple times as short as five seconds.

Cost Reduction: The proposed technology will reduce the overall cost of clean-up operations. Costs for chemical analysis will be reduced because the whole process from sample preparation to data management can be automated and performed onsite. Operational cost benefits will result from the availability of real-time analysis. Manpower and equipment costs would be reduced through the use of the robot operated chemical instrumentation due to improved operational efficiency.

Reduction of Heslth Risks: The automation of sample preparation steps and actual site mapping would eliminate somple bandling by laboratory personnel. Further, survey worker exposure to hazardous locations would be significantly reduced or eliminated. Insurance premiums alone will be reduced (it should be cheaper to insure robots than workers).

Reduction of Environmental Risks: Onsite, real-time analysis with the robot operated analyzer would provide improved site monitoring during decontamination. Using teal-time analysis, engineers could monitor and respono to problems in less time than is possible with conventional chemical analysis.

Improved Operations: The robot operated analysis instrumentation would improve the capabilities of DOE response in each of the three phases of remedial efforts by providing a more efficient. streanlined beginning-to-end operational capability. During the survey state worker exposure is reduced; and mapping and characterization cfforts proceed efficiently with the aid of real-time, remote controlled mapping and analysis. For example, when mapping out the contaminated area, engineers could review analytical results immediately and direct more intensive efforts where they are needed most. More accurate and otailed characterization of the waste site would concentrate restoration efforts to contaninated areas and eliminate needless efforts on areas that are in compliance or pose minimum risk. Once decontamination efforts are begun, removed materials and remaining site locations could be continually monitored to measure the progress of clean-up operations. Remediation operations would proceed faster because field engineers could have near realtime results indicating the status with respect to regulatory requirements.

Waste Minimization: Real-time monitoring of decontamisation operations would have a direct impact on the amount of waste generated diring remediation. Procedures could be halted as soon as compliance is achieved, preventing excess of temoval of otherwise clean material. More detailed mapping and characterization of site would identify areas that are in compliance and do not require decontamination. The analyses would be able to classify materials as they are removed thus optimizing the handling requirements to be consistent with their hazard level.

\section{CLR 3D Mapper Advantages over all Other Carrent Technologies}

Immunity to Ambient Light and Surtace Shading: The FM CLR is immune to ambient lighting conditions in an exactly anatogous way that FM radio is immune to the background amplitude bursts in Jightning. The other methods depend upon amplitude detection.

Lightweight Radiation Resistant Fiber Optic Iniplementation: The high sensitivity of the FM CLR detection process allows the use of a remote scanner at the end of an optical fiber with no solid state electronics. Becanse of the high efficiency of the optical fibers, the remote scanner could, in principle, be kilometers fron the integrated workstation. 
Benefits of the CLR 3D Mapper Performance Advantage: The fast CLR 3D Mapping capability will not impede or interrupt D\&D operations. Immunity to lighting conditions means that other D\&D operations will not have to be distapted while lighting is controlled for 3D mapping operations. A more accurate 3D mapping capability allows robotic operations to proceed with less supervision for characterization, decontamination, and dismantling operations. Characterization benefits from the high accuracy CLR 3D mapping are:

- Allows clear visualization of surface and more accurate planning and ex ecution of surface contaminant map ping operations

- Allows the sensor probe to follow a surface at close proximity without collision

- Supports object shape and texture de termination and better contarninant penetration modeling

\subsection{Fature Developments}

The Phase III 3D-ICAS development is aimed at demonstrating an fielđable contamination 30 mapping, aralysis and archiving system to support D\&D operations. Highlights of the 3D-ICAS system capability and performance improvements to be accomplished in Phase II are as follows:

- Entegration of the multisensor probe and supporting robot arm 3D-ICAS sensor subsystem) with a DOD pro vided mobility platform

- Integration of the CLR 3D Mapper subsystem with a DOE provided mobility platform
- Reduction in size and weight and field hardening of sensor subsystems and analysis hardware

- Improvement in speed of the CLR guided 6DOF multisensor probe end effoctor tracking and control

- Increase in flexibility of sensor route planning to allow a variety of map ping coverages for each sensor com ponent

- Refinement of contaminant detection and analysis software for all sensor components

- Refinement of system control, dis play, and other human interface soft ware

- Adaptation of 3D-ICAS system mis sion to evolving DOE site cleanup re quirtments

Beyond the 3D-ICAS development effort the seasor subsystem components may be adapted to other DOE applications. For example the sensor components may be applied to a floor survey robot such as MAX developed by Savannah River Technology Center. The MVS sensor may be applied to identify mixed waste chemical components stored in glass or transparent plastic containers.

The CLR 3D mapping tectimology will be extended in perfornance by other govemnent and industrial development applications to both higher speed and greater accuracy for robotics and metrology applications. A radiation hardened CLR 3D Mapper is being designed as a Remote Metrology Subsystem for the International Thermonuciear Reactor Subsystem. This development will take advantage of the compact no-moving-parts scanner 
technology development by CRC now being sponsored by METC under contract DE-AC21$94 \mathrm{MC} 31190$.

\subsection{References}

1. Phase I Topical Report to DOE. 1994. 3Dimensional Integrated Characterizaiton and Archiving System (3D-ICAS).

2. D.A. Long. 1977. Raman Spectroscopy, McGraw-Hill, New York.

3. HL Ower, J.M. Tedesco, and J.B. Slater. December 27, 1994. (Kaiser Optical Systems, Ann Arbor, MI), Remote Optical Measurement Probe, US Patent 5377004.

4. T. Lopez, F.R. Reich, and J.G. Douglas. Summary of FY 1994. Raman Spectroscopy Technology Cold Test Activities Document, US DOE Contract DE-AC06-87RLI0930, 4-8 (1995) available as Westinghouse-Hanford Document WHC-SE-WM-RPT-1 16, Westinghouse-Hanford, Rjchland, Washington.

5. K.P.J. Willams, J. Raman Spectrosc., 21 $147(1990)$.

6. J. Sawatski, Proc.XIII Internatinal Conference on Raman Spectroscopy (ICORS XIII), Eds. W. Keifer, G. Schaack, F.W. Schneider, and H.W. Schrotter, Additional Presenations Volume, Wiley, Chichester (1992).

7. T.F. Conney, H.T. Skinner, and S.M. Angel. 1995. Personal Communication.

8. R.D. MeLachlan, G.L. Jewett, and J.C. evans. 1986. (Dow Chemical Co., Midland, MI), Fiber-Optic Probe for Senstive Raman Analysis, US Patent 453771. 